\section{Post-transplant cyclophosphamide versus anti-thymocyte globulin for graft-versus-host disease prevention in haploidentical transplantation for adult acute lymphoblastic leukemia}

\author{
Arnon Nagler, ${ }^{1 *}$ Abraham S. Kanate, ${ }^{2^{*}}$ Myriam Labopin, ${ }^{3 *}$ Fabio Ciceri, ${ }^{4}$ \\ Emanuele Angelucci, ${ }^{5}$ Yener Koc, ${ }^{6}$ Zafer Gülbas, ${ }^{7}$ William Arcese, ${ }^{8}$ Johanna \\ Tischer, ${ }^{9}$ Pietro Pioltelli, ${ }^{10}$ Hakan Ozdogu ${ }^{11}$ Boris Afanasyev, ${ }^{12}$ Depei $\mathbf{W u},{ }^{13}$ \\ Mutlu Arat, ${ }^{14}$ Zinaida Peric,${ }^{15}$ Sebastian Giebel, ${ }^{16}$ Bipin Savani ${ }^{17}$ and Mohamad \\ Mohty ${ }^{18}$
}

${ }^{1}$ Chaim Sheba Medical Center, Tel Aviv University, Tel-Hashomer, Israel; ${ }^{2}$ West Virginia University, Morgantown, WV, USA; ${ }^{3}$ Department of Hematology and EBMT Paris study office / CEREST-TC, Saint Antoine Hospital, Paris, France; ${ }^{4}$ Ospedale San Raffaele s.r.l., Milano, Italy; ${ }^{5}$ Ospedale San Martino, Department of Hematology II, Genova, Italy; ${ }^{6}$ Medical Park Hospitals, Stem Cell Transplant Unit, Antalya, Turkey; ${ }^{7}$ Anadolu Medical Center Hospital, Bone Marrow Transplantation Department, Kocaeli, Turkey; ${ }^{8 *}$ Tor Vergata" University of Rome, Stem Cell Transplant Unit, Policlinico Universitario Tor Vergata, Rome, Italy; ${ }^{~ K}$ Klinikum Grosshadern, Med. Klinik III, Munich, Germany;

${ }^{10}$ Ospedale San Gerardo, Clinica Ematologica dell'Universita Milano-Biocca, Monza, Italy; ${ }^{11}$ Baskent University Hospital, Hematology Division, Bone Marrow Transplantation Unit, Adana, Turkey; ${ }^{12}$ First State Pavlov Medical University of St. Petersburg, Raisa Gorbacheva Memorial Research Institute for Pediatric Oncology, Hematology, and Transplantation, St. Petersburg, Russia; ${ }^{13}$ First Affiliated Hospital of Soochow University, Department of Hematology, Suzhou, China; ${ }^{14}$ Florence Nightingale Sisli Hospital, Hematopoietic Stem Cell Transplantation Unit, Istanbul, Turkey; ${ }^{15}$ University Hospital Center Zagreb, School of Medicine, University of Zagreb, Zagreb, Croatia; ${ }^{16}$ Department of Bone Marrow Transplantation and Oncohematology, Maria Sklodowska-Curie Memorial Cancer Center and Institute of Oncology, Gliwice, Poland; ${ }^{17}$ Department of Hematology-Oncology, Vanderbilt University Medical Center, Nashville, TN, USA and ${ }^{18}$ Department of Hematology and EBMT Paris study office / CEREST-TC, Saint Antoine Hospital, INSERM UMR 938 and Université Pierre et Marie Curie, Paris, France

${ }^{\star} A N, A S K$ and $M L$ contributed equally as co-first authors.
Haematologica 2021

Volume 106(6):1591-1598

\section{ABSTRACT}

G raft-versus-host disease (GvHD) prophylaxis for unmanipulated haploidentical hematopoietic cell transplantation includes posttransplant cyclophosphamide (PTCy) and anti-thymocyte globulin (ATG). Utilizing data in the European Society for Blood and Marrow Transplantation registry, we compared ATG- versus PTCy-based GvHD prophylaxis in 434 adults with acute lymphoblastic leukemia undergoing haploidentical hematopoietic cell transplantation. Of the 434 patients included in this study, ATG was used in 98 and PTCy in $336 .$. The median follow-up was approximately 2 years. The baseline characteristics of the patients were similar between the groups except that the ATG group was more likely to have had relapsed/refractory acute lymphoblastic leukemia $(P=0.008)$, had conditioning not including total body irradiation $(P<0.001)$, have had peripheral blood as the source of their grafts $(P \leq 0.001)$ and to have been transplanted in an earlier timeperiod (median year of transplantation: 2011 vs. 2015). The 100-day rates of grade II-IV and III-IV acute GvHD were similar in the ATG and PTCy groups, as were 2-year chronic GvHD rates. On multivariate analysis, leukemia-free survival and overall survival were better with PTCy than with ATG prophylaxis. Relapse incidence was lower in the PTCy group $(P=0.03)$, while non-relapse mortality was not different. Advanced disease and lower performance score were associated with poorer leukemia-free survival and overall survival and advanced disease
(1598)

\section{Correspondence:}

ABRAHAM S. KANATE

askanate@hsc.wvu.edu

Received: January 12, 2020.

Accepted: April 28, 2020.

Pre-published: April 30, 2020.

https://doi.org/10.3324/haematol.2020.247296

(C)2021 Ferrata Storti Foundation

Material published in Haematologica is covered by copyright. All rights are reserved to the Ferrata Storti Foundation. Use of published material is allowed under the following terms and conditions:

https://creativecommons.org/licenses/by-nc/4.0/legalcode. Copies of published material are allowed for personal or internal use. Sharing published material for non-commercial purposes is subject to the following conditions:

https://creativecommons.org/licenses/by-nc/4.0/legalcode, sect. 3. Reproducing and sharing published material for commercial purposes is not allowed without permission in writing from the publisher. 
was associated with inferior GvHD-free/relapse-free survival. Compared to bone marrow grafts, peripheral grafts were associated with higher rates of GvHD. In patients with acute lymphoblastic leukemia undergoing unmanipulated haploidentical hematopoietic cell transplantation, PTCy for GvHD prevention resulted in a lower incidence of relapse and improved leukemia-free survival and overall survival, compared to ATG.

\section{Introduction}

Despite significant advances in the management of adult acute lymphoblastic leukemia (ALL), disease relapse remains a significant impediment to long-term leukemiafree survival, ${ }^{1}$ especially in adult patients aged $>20$ years and in those with advanced (relapsed/refractory) disease. ${ }^{2}$ Although fraught with challenges of disease relapse and non-relapse mortality, allogeneic hematopoietic cell transplantation (HCT) is a potentially curative option for these patients, and is often considered in high-risk and advanced ALL. ${ }^{2-4}$ In the absence of a suitable HLAmatched donor, allogeneic transplantation from a related haploidentical donor can be considered and such donors are a readily available source of grafts for most patients irrespective of racial/ethnic background. Indeed, the use of haploidentical HCT has increased steadily over the years in various hematologic malignancies including acute leukemia. ${ }^{4,5}$ To mitigate the risk of greater HLA-disparity and resultant graft rejection and graft-versus-host disease (GvHD) which were seen with haploidentical HCT, T-cell depletion was used historically, but this strategy was associated with higher risks of non-relapse mortality, disease relapse and delayed immune reconstitution. ${ }^{6.9}$

The use of unmanipulated, T-cell-replete grafts has revived haploidentical transplantation. Immunosuppression using anti-thymocyte globulin (ATG) in this setting has shown favorable results. ${ }^{10,11}$ Wang et al. conducted a biologically randomized trial, specifically in ALL patients in first complete remission, comparing matched sibling donor versus haploidentical donor transplantation using ATG-based GvHD prophylaxis and reported a similar 3-year leukemiafree survival $(60 \%$ vs. $61 \%$ by the intention-to-treat analysis). The administration of post-transplant cyclophosphamide (PTCy) after an unmanipulated haploidentical allograft has shown favorable results and has become widely utilized in the past decade. ${ }^{12,13}$ Registry data also support unmanipulated haploidentical HCT as a viable treatment option for ALL patients. ${ }^{14,15}$

Although both strategies are effective as GvHD prophylaxis, there is a paucity of comparative data on ATG versus PTCy in haploidentical transplantation. Ruggeri et al. conducted a retrospective study using data from the European Society for Blood and Marrow Transplantation (EBMT) registry on 308 patients with acute myeloid leukemia, and compared outcomes between those given ATG $(n=115)$ or PTCy $(n=193)$ as a GvHD prevention strategy. On multivariate analysis, compared to ATG, PTCy use was associated with significantly better leukemia-free survival $(P=0.03)$ and GvHD-free/relapsefree survival (GRFS) $(P=0.03)$. To our knowledge, no studies have reported comparative data between ATG and PTCy platforms in ALL patients undergoing haploidentical transplantation. ${ }^{16}$ We used the EBMT database to conduct a comparative analysis between ATG and PTCy strategies in ALL patients undergoing haploidentical HCT using bone marrow or peripheral blood as the source of hematopoietic cells for the graft.

\section{Methods}

\section{Data source and patients}

This is a retrospective multicenter analysis using the dataset of the Acute Leukemia Working Party of the EBMT group registry. The EBMT is a voluntary working group of more than 600 transplant centers that are required to report, annually, all consecutive hematopoietic cell transplants and follow-ups. Audits are performed routinely to determine the accuracy of the data. The study was planned and approved by the Acute Leukemia Working Party of the EBMT. In addition, the study protocol was approved by the institutional review board at each site and complied with countryspecific regulatory requirements. The study was conducted in accordance with the Declaration of Helsinki and Good Clinical Practice guidelines.

The subjects included in this analysis were adults ( $\geq 18$ years) with ALL who underwent their first haploidentical HCT between 2007 and 2017, were reported to the "Promise" database of the EBMT and received either ATG or PTCy as a GvHD prevention strategy. Recipients of haploidentical transplantation (mismatched by at least two or more HLA-loci to donors) received unmanipulated, bone marrow or peripheral blood grafts with additional GvHD prophylaxis, which consisted predominantly of a calcineurin inhibitor plus mycophenolate mofetil or a calcineurin inhibitor plus methotrexate. Patients who received grafts that had been manipulated ex vivo (T-cell-depleted or CD34-selected grafts) or who received both ATG and PTCy $(n=37)$ were excluded.

\section{Study endpoints and definitions}

The primary endpoint for this study was leukemia-free survival. Secondary endpoints were acute GvHD, chronic GvHD, relapse incidence, non-relapse mortality, GRFS and overall survival. Refined GRFS was defined as survival without the following events: grade III or IV acute GvHD, severe chronic GvHD, disease relapse, or death from any cause after haploidentical HCT..$^{17,18}$ Leukemia-free survival was calculated until the date of first relapse, death from any cause or the last follow-up for patients alive in complete remission. Relapse was defined as disease recurrence and appearance of blasts in the peripheral blood or bone marrow ( $>5 \%$ ) after having achieved complete remission. Nonrelapse mortality was defined as death from any cause other than relapse. Acute GvHD was graded according to the modified Seattle Glucksberg criteria ${ }^{19}$ and chronic GvHD according to the revised Seattle criteria. ${ }^{20}$ The conditioning regimen was defined as myeloablative when it contained total body irradiation (TBI) at a dose $>6$ Gray or a total dose of busulfan $>8 \mathrm{mg} / \mathrm{kg}$ (orally) or $>6.4$ $\mathrm{mg} / \mathrm{kg}$ (intravenously). All other conditioning regimens were defined as reduced intensity. ${ }^{21}$ Neutrophil engraftment was defined as the first of 3 successive days with an absolute neutrophil count $\geq 500 / \mu \mathrm{L}$.

\section{Statistical analysis}

Patient-, disease-, and transplant-related characteristics for the 
two cohorts (reducing intensity/myeloablative conditioning) were compared using $\chi^{2}$ statistics for categorical variables and the Mann-Whitney test for continuous variables. Survival statistics (leukemia-free survival, overall survival and GRFS) were estimated by the Kaplan-Meier method. Cumulative incidence functions were used to estimate neutrophil engraftment, acute GvHD, chronic GvHD, relapse incidence and non-relapse mortality. Competing risks were death for engraftment and relapse incidence, relapse for non-relapse mortality, and relapse or death for acute and chronic GvHD. Univariate analysis (Online Supplementary Table S1) was carried out using the log-rank test for GRFS, overall survival and leukemia-free survival, and the Gray test for cumulative incidence functions. A Cox proportional hazards model was used for multivariate regression. All variables differing significantly between the two groups or factors associated with one outcome in univariate analysis were included in the Cox model. To test for a center effect, we introduced a random effect or frailty for each center into the model..$^{22,23}$ Results are expressed as a hazard ratio (HR) with a $95 \%$ confidence interval $(95 \% \mathrm{CI})$. All tests were two-sided. The type I error rate was fixed at 0.05 for the determination of factors associated with time-to-event outcomes. Statistical analyses were performed with SPSS 24.0 (SPSS Inc, Chicago, IL, USA) and R 3.4.0 (R Core Team [2017]. R: A language and environment for statistical computing. R Foundation for Statistical Computing, Vienna, Austria. URL https://www.Rproject.org/).

\section{Results}

\section{Baseline characteristics}

In all, 434 patients undergoing haploidentical HCT for ALL were included in the study, which comprised two groups divided according to which GvHD prophylaxis the patients received: ATG $(n=98)$ or PTCy $(n=336)$. Baseline patient-, disease- and transplantation-related characteristics are shown in Table 1. There were no significant differences between the groups in terms of patients' age, gender, ALL subtype, Karnofsky Performance Score <90, HCT-Comorbidity Index $\geq 3$, donors' age and donor-recipient combinations of sex and cytomegalovirus serological status. ATG recipients were more likely to have relapsed/refractory ALL compared to PTCy recipients ( $30.6 \%$ vs. $16.4 \%$, respectively; $P=0.008$ ). Although the difference was not statistically significant, myeloablative conditioning regimens were given to a greater proportion of PTCy-treated patients than ATG-treated patients (78.3\% vs. $69.4 \%$, respectively; $P=0.07$ ). The PTCy group was more likely to have received TBI $(45.2 \%$ vs. $26.5 \%$; $P \leq 0.001)$. A TBI dose of $\geq 10$ Gy was administered to $25.8 \%$ of the patients in the PTCy group and $14.3 \%$ of those in the ATG group. Bone marrow was the graft source in $52.1 \%$ and $31.6 \%$ of patients in the PTCy and ATG groups, respectively $(P \leq 0.001)$. ATG-based haploidentical transplants were carried out during an earlier period (median year of transplant, 2011) compared to PTCy-based transplants (median year of transplant, 2015) $(P \leq 0.0001)$. The median follow-up for survivors in the ATG and PTCy groups was 55 months (range, 14-79) and 22 months (range, 12-37), respectively.

\section{Engraftment}

The cumulative incidence of engraftment at day 60 was $91.7 \%$ (95\% CI: 83.7-95.8) and $92.5 \%$ (95\% CI: 89-95) in the ATG and PTCy groups, respectively $(P=0.11)$.
Table 1. Baseline patient-, donor- and transplant-related characteristics in the entire cohort of haploidentical donor transplant recipients and groups stratified by graft-versus-host disease prevention strategy.

\begin{tabular}{|c|c|c|c|c|}
\hline Baseline variable & $\begin{array}{l}\text { All patients } \\
N=434\end{array}$ & ATC, $N=98$ & $\begin{array}{l}\text { GVHD prophyl } \\
\text { PTCy, N=336 }\end{array}$ & $\begin{array}{l}\text { axis } \\
\text { P-valu }\end{array}$ \\
\hline $\begin{array}{l}\text { Recipients'age in years, } \\
\text { median (range) }\end{array}$ & $35.6(18-76)$ & $35.5(18-76)$ & $36(18-73)$ & 0.93 \\
\hline Male recipient*, n (\%) & $274(63)$ & $61(62.2)$ & $213(63.8)$ & 0.78 \\
\hline $\begin{array}{l}\text { ALL subtype, n (\%) } \\
\text { Ph-negative B-ALL } \\
\text { Ph-positive B-ALL } \\
\text { T-ALL }\end{array}$ & $\begin{array}{l}154(35.4) \\
140(32.3) \\
140(32.3)\end{array}$ & $\begin{array}{l}34(34.7) \\
36(36.7) \\
28(28.6)\end{array}$ & $\begin{array}{l}120(35.7) \\
104(31.0) \\
112(33.3)\end{array}$ & 0.51 \\
\hline $\begin{array}{l}\text { Remission status, n (\%) } \\
\text { CR1 } \\
\text { CR2 or beyond } \\
\text { Advanced (r/r) }\end{array}$ & $\begin{array}{l}208(47.9) \\
141(32.5) \\
85(19.6)\end{array}$ & $\begin{array}{l}41(41.8) \\
27(27.6) \\
30(30.6)\end{array}$ & $\begin{array}{l}167(49.7) \\
114(33.9) \\
55(16.4)\end{array}$ & 0.008 \\
\hline $\begin{array}{l}\text { KPS, n (\%) } \\
\quad \geq 90 \\
\quad<90 \\
\text { Missing }\end{array}$ & $\begin{array}{l}293(72) \\
114(28) \\
27\end{array}$ & $\begin{array}{c}64(65.3) \\
23(23.5) \\
11\end{array}$ & $\begin{array}{c}229(68.2) \\
91(27.0) \\
16\end{array}$ & 0.71 \\
\hline $\begin{array}{l}\text { Comorbidity Index, n (\%) } \\
\leq 2 \\
\geq 3 \\
\text { Missing }\end{array}$ & $\begin{array}{c}188(79) \\
50(21) \\
196\end{array}$ & $\begin{array}{c}27(71.1) \\
11(28.9) \\
60\end{array}$ & $\begin{array}{c}161(80.5) \\
39(19.5) \\
136\end{array}$ & 0.19 \\
\hline $\begin{array}{l}\text { Prior autologous } \\
\text { transplantation, n (\%) }\end{array}$ & $21(4.8)$ & $5(5)$ & $16(4.8)$ & 0.8 \\
\hline $\begin{array}{l}\text { Conditioning intensity, n (\%) } \\
\text { Myeloablative } \\
\text { Reduced intensity }\end{array}$ & $\begin{array}{l}331(76.3) \\
103(23.7)\end{array}$ & $\begin{array}{l}68(69.4) \\
30(30.6)\end{array}$ & $\begin{array}{l}263(78.3) \\
73(21.7)\end{array}$ & 0.07 \\
\hline Total body irradiation, n (\%) & $178(41)$ & $26(26.5)$ & $152(45.2)$ & $<0.00$ \\
\hline $\begin{array}{l}\text { Additional GvHD prophylaxis, } \\
\text { Cyclosporine/MTX } \\
\text { Cyclosporine/MMF } \\
\text { Tacrolimus/MMF } \\
\text { Sirolimus/MMF } \\
\text { Cyclosporine/MMF/ } \\
\text { MTX } \\
\text { Cyclosporine } \\
\text { Tacrolimus } \\
\text { Tacrolimus/sirolimus } \\
\text { MMF } \\
\text { Sirolimus } \\
\text { Tacrolimus/MMF/MTX } \\
\text { Missing }\end{array}$ & $\begin{array}{l}(\%) \\
23(5.4) \\
205(47.3) \\
88(20.6) \\
29(6.8) \\
45(10.5)\end{array}$ & $\begin{array}{c}14(14.9) \\
4(4.3) \\
3(3.2) \\
21(22.3) \\
40(42.5)\end{array}$ & $\begin{array}{c}9(2.7) \\
201(59.8) \\
85(25.5) \\
8(2.4) \\
5(1.5) \\
\\
10(3) \\
9(2.7) \\
3(0.9) \\
3(0.9) \\
0 \\
0 \\
3\end{array}$ & NA \\
\hline
\end{tabular}

\section{Graft source, $n(\%)$}

Bone marrow

Peripheral blood

Donors' age in years, median (range)

\begin{tabular}{|c|c|c|c|c|}
\hline Female D $\rightarrow$ male R*, n (\%) & $122(28.2)$ & $30(30.6)$ & $92(27.5)$ & 0.55 \\
\hline $\begin{array}{l}\text { D7R CMV status, n (\%) } \\
\text { Negative/negative } \\
\text { Positive/negative } \\
\text { Negative/positive } \\
\text { Positive/positive } \\
\text { Missing }\end{array}$ & $\begin{array}{c}57(13.5) \\
39(9.3) \\
59(14) \\
266(63.2) \\
13\end{array}$ & $\begin{array}{c}19(20.2) \\
9(9.6) \\
12(12.8) \\
54(57.5) \\
4\end{array}$ & $\begin{array}{c}38(11.6) \\
30(9.2) \\
47(14.4) \\
212(64.8) \\
9\end{array}$ & 0.19 \\
\hline $\begin{array}{l}\text { Year of transplant, } \\
\text { median (range) }\end{array}$ & $\begin{array}{c}2015 \\
(2007-2017)\end{array}$ & $\begin{array}{c}2011 \\
(2007-2017)\end{array}$ & $\begin{array}{c}2015 \\
(2008-2017) 1\end{array}$ & $<0.000$ \\
\hline $\begin{array}{l}\text { Follow-up in months, median } \\
\text { (range) }\end{array}$ & $24.4(12-40)$ & $55(14-79)$ & $22(12-37)$ & \\
\hline
\end{tabular}

*Data on sex missing for two patients. GvHD: graft-versus-host disease; ATG: anti-thymocyte globulin; PTCy: post-transplant cyclophosphamide;ALL: acute lymphoblastic leukemia; Ph: Philadelphia chromosome; CR1: first complete remission; CR2: second complete remission; $r / r$ r relapsed/refractory; KPS: Karnofsky Performance Score; MTX: methotrexate; MMF: mycophenolate mofetil; NA: not available; D: donor; R: recipient; CMV: cytomegalovirus. 


\section{Acute and chronic graft-versus-host disease}

The cumulative incidence of grade II-IV acute GvHD at day 100 (Table 2) in the ATG group was $32.7 \%$ (95\% CI: 23.4-42.3) compared to $30.5 \%$ (95\% CI: $25.5-35.6$ ) in the PTCy group $(P=0.37)$. The corresponding rates of severe (grades III-IV) acute GVHD were $11.6 \%$ (95\% CI: 6.1 18.9) and $14.1 \%$ (95\% CI: $10.6-18.2)$ in the ATG and PTCy groups respectively $(P=0.56)$. On multivariate analy-

Table 2. Post-transplant outcomes (unadjusted) by graft-versus-host disease prevention strategy.

\begin{tabular}{lccc}
\hline Post-transplant outcomes* & AIG (95\% CI) & PiCy (95\% CI) & P-value \\
\hline Engraftment & $91.7 \%(83.7-95.8)$ & $92.5 \%(89-95)$ & 0.11 \\
Acute GvHD II-IV & $32.7 \%(23.4-42.3)$ & $30.5 \%(25.5-35.6)$ & 0.37 \\
\hline Acute GvHD III-IV & $11.6 \%(6.1-18.9)$ & $14.1 \%(10.6-18.2)$ & 0.56 \\
Chronic GvHD & $27.7 \%(17.8-38.5)$ & $31.7 \%(26.1-37.4)$ & 0.58 \\
\hline Extensive chronic GvHD & $7.8 \%(3.1-15.2)$ & $12.1 \%(8.4-16.5)$ & 0.37 \\
Relapse incidence & $43 \%(32-53.5)$ & $33.8 \%(28.1-39.5)$ & 0.11 \\
\hline Non-relapse mortality & $32.9 \%(23.1-43.1)$ & $26.7 \%(21.8-31.8)$ & 0.23 \\
Leukemia free survival & $24.1 \%(14.5-33.8)$ & $39.6 \%(33.6-45.5)$ & 0.007 \\
\hline Overall survival & $27.4 \%(17.4-37.3)$ & $48.4 \%(42.3-54.6)$ & 0.001 \\
GvHD/relapse-free survival & $20 \%(10.9-29.1)$ & $31.8 \%(26.2-37.5)$ & 0.04
\end{tabular}

*All outcomes are at 2 years except for acute graft-versus-host disease which is at 100 days after transplantation.ATG: anti-thymocyte globulin; PTCy: post-transplant cyclophosphamide; $95 \% \mathrm{CI}$ : 95\% confidence interval; GvHD: graft-versus-host disease.

Table 3. Multivariate analysis of post-transplant outcomes and baseline variables.

\begin{tabular}{|c|c|c|c|c|c|c|c|c|c|c|c|c|c|c|}
\hline $\begin{array}{l}\text { Post-transplant } \\
\text { outcomes }\end{array}$ & $\begin{array}{c}\text { t PICy vs. } \\
\text { ATG }\end{array}$ & $\begin{array}{l}\mathrm{Ph}^{+} \\
\text {B-ALL }\end{array}$ & T-ALL & $\begin{array}{c}\text { Age } \\
\text { (per } \\
10 \text { years) }\end{array}$ & $\geq \mathrm{CR} 2$ & Advanced & $\begin{array}{l}K P S \\
\geq 90\end{array}$ & $\begin{array}{l}\text { RIC vs. } \\
\text { MAC }\end{array}$ & $\begin{array}{l}\text { PB vs. } \\
\text { BM }\end{array}$ & $\begin{array}{l}\text { Female } \\
\text { (D) } \rightarrow \\
\text { male (B) }\end{array}$ & $\begin{array}{l}\text { CMV } \\
(\mathrm{R})+\end{array}$ & $\begin{array}{l}\text { CMV } \\
\text { (D)- }\end{array}$ & $\begin{array}{l}\text { TBI vS. } \\
\text { chemo }\end{array}$ & Center \\
\hline \multicolumn{15}{|l|}{ Relapse } \\
\hline $\begin{array}{l}\text { HR } \\
(95 \% \mathrm{Cl})\end{array}$ & $\begin{array}{c}0.61 \\
(0.39-0.94)\end{array}$ & $\begin{array}{c}0.66 \\
(0.39-1.12)\end{array}$ & $\begin{array}{c}1.05 \\
(0.69-1.6)\end{array}$ & $\begin{array}{c}0.97 \\
(0.83-1.14)\end{array}$ & $\begin{array}{c}2.06 \\
(1.32-3.21)\end{array}$ & $\begin{array}{c}4.99 \\
(3.13-7.93)\end{array}$ & $\begin{array}{c}0.98 \\
(0.65-1.48)\end{array}$ & $\begin{array}{c}1.08 \\
(0.67-1.74)\end{array}$ & $\begin{array}{c}0.971 \\
(0.66-1.44)\end{array}$ & $\begin{array}{c}0.84 \\
(0.56-1.27)\end{array}$ & $\begin{array}{c}1.14 \\
(0.70-1.87)\end{array}$ & $\begin{array}{c}0.92 \\
(0.59-1.43)\end{array}$ & $\begin{array}{c}0.97 \\
(0.67-1.42)\end{array}$ & \\
\hline$P$-value & 0.03 & 0.12 & 0.81 & 0.72 & 0.001 & $<10-5$ & 0.93 & 0.74 & 0.88 & 0.42 & 0.59 & 0.7 & 0.88 & 0.39 \\
\hline \multicolumn{15}{|l|}{ NRM } \\
\hline $\begin{array}{l}\mathrm{HR} \\
(95 \% \mathrm{Cl})\end{array}$ & $\begin{array}{c}0.68 \\
(0.42-1.11)\end{array}$ & $\begin{array}{c}1.26 \\
(0.76-2.1)\end{array}$ & $\begin{array}{c}1.04 \\
(0.62-1.74)\end{array}$ & $\begin{array}{c}1.1 \\
(0.94-1.3)\end{array}$ & $\begin{array}{c}1.71 \\
(1.07-2.72)\end{array}$ & $\begin{array}{c}1.82 \\
(1.02-3.24)\end{array}$ & $\begin{array}{c}0.46 \\
(0.30-0.7)\end{array}$ & $\begin{array}{c}0.97 \\
(0.59-1.6)\end{array}$ & $\begin{array}{c}1.48 \\
(0.97-2.26)\end{array}$ & $\begin{array}{c}0.76 \\
(0.47-1.22)\end{array}$ & $\begin{array}{c}0.90 \\
(0.53-1.55)\end{array}$ & $\begin{array}{c}1.33 \\
(0.8-2.23)\end{array}$ & $\begin{array}{c}0.59 \\
(0.38-0.92)\end{array}$ & \\
\hline$P$-value & 0.12 & 0.37 & 0.88 & 0.24 & 0.02 & 0.04 & 0.0003 & 0.91 & 0.07 & 0.25 & 0.71 & 0.27 & 0.02 & 0.43 \\
\hline \multicolumn{15}{|l|}{ LFS } \\
\hline $\begin{array}{l}\text { HR } \\
(95 \% \mathrm{CI})\end{array}$ & $\begin{array}{c}0.67 \\
(0.46-0.97)\end{array}$ & $\begin{array}{c}0.94 \\
(0.63-1.35)\end{array}$ & $\begin{array}{c}1.06 \\
(0.76-1.5)\end{array}$ & $\begin{array}{c}1.04 \\
(0.93-1.17)\end{array}$ & $\begin{array}{c}1.91 \\
(1.37-2.66)\end{array}$ & $\begin{array}{c}3.35 \\
(2.3-4.88)\end{array}$ & $\begin{array}{c}0.68 \\
(0.5-0.93)\end{array}$ & $\begin{array}{c}1.06 \\
(0.74-1.52)\end{array}$ & $\begin{array}{c}1.06 \\
(0.76-1.46)\end{array}$ & $\begin{array}{c}0.8 \\
(0.58-1.1)\end{array}$ & $\begin{array}{c}0.95 \\
(0.65-1.39)\end{array}$ & $\begin{array}{c}1.11 \\
(0.78-1.57)\end{array}$ & $\begin{array}{c}0.79 \\
(0.58-1.07)\end{array}$ & \\
\hline$P$-value & 0.03 & 0.69 & 0.72 & 0.48 & 0.0001 & $<10-5$ & 0.01 & 0.75 & 0.75 & 0.16 & 0.81 & 0.56 & 0.13 & 0.16 \\
\hline \multicolumn{15}{|l|}{ OS } \\
\hline $\begin{array}{l}\text { HR } \\
(95 \% \mathrm{CI})\end{array}$ & $\begin{array}{c}0.6 \\
(0.42-0.84)\end{array}$ & $\begin{array}{c}0.92 \\
(0.63-1.35)\end{array}$ & $\begin{array}{c}1.06 \\
(0.75-1.49)\end{array}$ & $\begin{array}{c}1.07 \\
(0.95-1.2)\end{array}$ & $\begin{array}{c}1.88 \\
(1.33-2.64)\end{array}$ & $\begin{array}{c}3.13 \\
(2.15-4.55)\end{array}$ & $\begin{array}{c}0.61 \\
(0.45-0.827)\end{array}$ & $\begin{array}{c}0.96 \\
(0.68-1.38)\end{array}$ & $\begin{array}{c}1.29 \\
(0.96-1.75)(0\end{array}$ & $\begin{array}{r}0.75 \\
.54-1.04)\end{array}$ & $\begin{array}{c}1.07 \\
(0.73-1.58)\end{array}$ & $\begin{array}{c}1.17 \\
(0.82-1.66)\end{array}$ & $\begin{array}{c}0.78 \\
(0.58-1.06)\end{array}$ & \\
\hline$P$-value & 0.003 & 0.67 & 0.75 & 0.26 & 0.0003 & $<10-5$ & 0.001 & 0.84 & 0.09 & 0.08 & 0.73 & 0.4 & 0.12 & 0.91 \\
\hline \multicolumn{15}{|l|}{ GRFS } \\
\hline $\begin{array}{l}\mathrm{HR} \\
(95 \% \mathrm{CI})\end{array}$ & $\begin{array}{c}0.79 \\
(0.57-1.11)\end{array}$ & $\begin{array}{c}0.88 \\
(0.62-1.24)\end{array}$ & $\begin{array}{c}1 \\
(0.72-1.35)\end{array}$ & $\begin{array}{c}1 \\
(0.90-1.11)\end{array}$ & $\begin{array}{c}1.42 \\
(1.05-1.92)\end{array}$ & $\begin{array}{c}2.45 \\
(1.73-3.47)\end{array}$ & $\begin{array}{c}0.77 \\
(0.58-1.02)\end{array}$ & $\begin{array}{c}0.94 \\
(0.68-1.31)\end{array}$ & $\begin{array}{c}1.18 \\
(0.89-1.57)\end{array}$ & $\begin{array}{c}0.97 \\
(0.73-1.31)\end{array}$ & $\begin{array}{c}1.05 \\
(0.75-1.49)\end{array}$ & $\begin{array}{c}1.08 \\
(0.79-1.49)\end{array}$ & $\begin{array}{c}0.87 \\
(0.66-1.15)\end{array}$ & \\
\hline$P$-value & 0.17 & 0.47 & 0.94 & 0.96 & 0.02 & $<10-5$ & 0.07 & 0.71 & 0.26 & 0.85 & 0.77 & 0.63 & 0.34 & 0.24 \\
\hline \multicolumn{15}{|c|}{ Acute GvHD II-IV } \\
\hline $\begin{array}{l}\mathrm{HR} \\
(95 \% \mathrm{CI})\end{array}$ & $\begin{array}{c}0.92 \\
(0.55-1.51)\end{array}$ & $\begin{array}{c}1.09 \\
(0.68-1.77)\end{array}$ & $\begin{array}{c}0.52 \\
(0.32-0.85)\end{array}$ & $\begin{array}{c}0.86 \\
(0.73-1.01)\end{array}$ & $\begin{array}{c}0.98 \\
(0.63-1.54)\end{array}$ & $\begin{array}{c}1.71 \\
(0.98-2.99)\end{array}$ & $\begin{array}{c}1.15 \\
(0.73-1.81)\end{array}$ & $\begin{array}{c}0.57 \\
(0.34-0.97)\end{array}$ & $\begin{array}{c}1.64 \\
(1.06-2.53)\end{array}$ & $\begin{array}{c}1.36 \\
(0.9-2.06)\end{array}$ & $\begin{array}{c}1.11 \\
(0.64-1.94)\end{array}$ & $\begin{array}{c}1.29 \\
(0.78-2.15)\end{array}$ & $\begin{array}{c}1.26 \\
(0.83-1.92)\end{array}$ & \\
\hline$P$-value & 0.73 & 0.72 & 0.008 & 0.06 & 0.94 & 0.06 & 0.55 & 0.04 & 0.03 & 0.14 & 0.72 & 0.32 & 0.28 & 0.12 \\
\hline \multicolumn{15}{|l|}{ chronic GvHD } \\
\hline $\begin{array}{l}\text { HR } \\
(95 \% \mathrm{CI})\end{array}$ & $\begin{array}{c}0.79 \\
(0.38-1.64)\end{array}$ & $\begin{array}{c}0.83 \\
(0.45-1.53)\end{array}$ & $\begin{array}{c}0.63 \\
(0.35-1.16)\end{array}$ & $\begin{array}{c}0.89 \\
(0.74-1.07)\end{array}$ & $\begin{array}{c}0.95 \\
(0.56-1.6)\end{array}$ & $\begin{array}{c}0.77 \\
(0.35-1.68)\end{array}$ & $\begin{array}{c}0.69 \\
(0.41-1.17)\end{array}$ & $\begin{array}{c}0.65 \\
(0.33-1.26)\end{array}$ & $\begin{array}{c}1.82 \\
(1.03-3.23)\end{array}$ & $\begin{array}{c}1.26 \\
(0.77-2.07)\end{array}$ & $\begin{array}{c}0.52 \\
(0.3-0.9)\end{array}$ & $\begin{array}{c}1.11 \\
(0.63-1.97)\end{array}$ & $\begin{array}{c}0.93 \\
(0.54-1.58)\end{array}$ & \\
\hline$P$-value & 0.52 & 0.55 & 0.14 & 0.22 & 0.83 & 0.51 & 0.17 & 0.20 & 0.04 & 0.35 & 0.02 & 0.71 & 0.77 & 0.02 \\
\hline
\end{tabular}

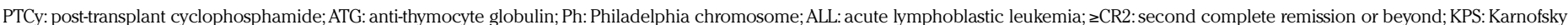

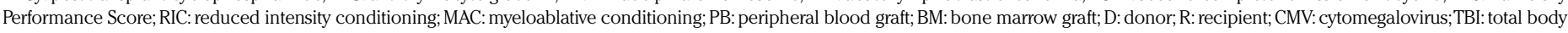
irradiation; NRM: non-relapse mortality; LFS: leukemia-free survival; OS: overall survival; GRFS: GvHD-free/relapse-free survival; GvHD: graft-versus-host disease. sis (Table 3) there was no difference in the risk of grade IIIV acute GVHD in the ATG group relative to the PTCy group (HR=0.92, 95\% CI: $0.55-1.51 ; \quad P=0.73)$. Independently of the GvHD prevention strategy, on multivariate analysis, the use of peripheral blood allografts GvHD (HR=1.64, 95\% CI: 1.06-2.53; $P=0.03)$, whereas a diagnosis of T-ALL (HR=0.52, 95\% CI: $0.32-0.85 ; P=0.008$ ) and reduced intensity conditioning ( $\mathrm{HR}=0.57,95 \% \mathrm{CI}$ : 0.34-0.97) were associated with a lower risk (Table 3).

The cumulative incidence of chronic GvHD at 2 years after haploidentical transplantation was $27.7 \%$ (95\% CI: 17.8-38.5) and $31.7 \%$ (95\% CI: 26.1-37.4) in the ATG and PTCy groups, respectively (Table 2). The corresponding incidences of extensive chronic GvHD were $7.8 \%(95 \%$ CI: $3.1-15.2)$ and $12.1 \%$ (95\% CI: 8.4-16.5), respectively $(P=0.37)$. Multivariate analysis (Table 3 ) showed no difference in chronic GvHD outcomes between the two groups ( $\mathrm{HR}=0.79$, 95\% CI: 0.38-1.64; $P=0.52$ ). The use of peripheral blood as the graft source was associated with a higher risk of chronic GvHD (HR=1.82, 95\% CI: 1.03-3.23; $P=0.04)$, independently of the GvHD prevention used. Recipient cytomegalovirus seropositive status was associated with a lower risk of chronic GvHD ( $\mathrm{HR}=0.52,95 \%$ CI: $0.3-0.9 ; P=0.02$ ).

\section{Non-relapse mortality and relapse}

Among the ALL patients who underwent haploidentical transplantation, the 2-year non-relapse mortality was was associated with higher rates of grade II-IV acute 
32.9\% (95\% CI: $23.1-43.1)$ and $26.7 \%$ (95\% CI: 21.8-31.8) in the ATG and PTCy groups, respectively $(P=0.23)$ (Table $2)$. On multivariate analysis, there was no significant difference in non-relapse mortality between the groups ( $\mathrm{HR}=0.68,95 \% \mathrm{CI}: 0.42-1.11 ; P=0.12$ ). Independently of whether patients received ATG or PTCy as GvHD prophylaxis, pre-transplant status of being in second or further complete remission or having advanced disease was associated with higher rates of non-relapse mortality (Table 3). A Karnofsky Performance Score $\geq 90$ was associated with a lower non-relapse mortality rate $(\mathrm{HR}=0.46$, $95 \%$ CI: $0.30-0.7 ; P=0.0003)$. Use of TBI in conditioning was associated with lower non-relapse mortality (HR=0.59, 95\% CI: 0.38-0.92; $P=0.02$ ) compared to regimens containing only chemotherapy.

The cumulative incidence of ALL relapse at 2 years was similar between the ATG and PTCy groups, being $43 \%$ (95\% CI: 32-53.5) and 33.8\% (95\% CI: 28.1-39.5), respectively $(P=0.11)$ (Table 2). On multivariate analysis, PTCy as GvHD prophylaxis was associated with a lower risk of relapse ( $\mathrm{HR}=0.61,95 \%$ CI: 0.39-0.94; $P=0.03$ ). Disease status., i.e. being in second complete remission or beyond $(\mathrm{HR}=2.06,95 \%$ CI: $1.32-3.21 ; P=0.001)$ or having advanced ALL ( $\mathrm{HR}=4.99,95 \%$ CI: 3.13-7.93; $\left.P<10^{-5}\right)$ were noted to be independent risk factors for post-transplant relapse (Table 3). Figure $1 \mathrm{~A}$ and $\mathrm{B}$ show the adjusted nonrelapse mortality and relapse incidence in the ATG and PTCy groups, respectively.

\section{Survival}

With a median follow-up of 24 months, the 2-year leukemia-free survival rates in the ATG and PTCy groups were $24.1 \%$ (95\% CI: $14.5-33.8)$ and $39.6 \%$ (95\% CI: 33.6-45.5), respectively ( $P=0.007$ ) (Table 2$)$. On multivariate analysis (Table 3 ), relative to the ATG group, patients in the PTCy group had a lower risk of therapy failure (the inverse of leukemia-free survival) ( $\mathrm{HR}=0.67,95 \% \mathrm{CI}$ : 0.46 $0.97 ; P=0.03)$. Independently of the $\mathrm{GvHD}$ prevention strategy, a disease status of second complete remission of beyond ( $\mathrm{HR}=1.91,95 \% \mathrm{CI}: 1.37-2.66 ; P=0.0001)$ and

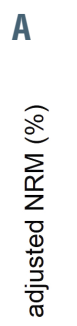

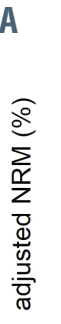

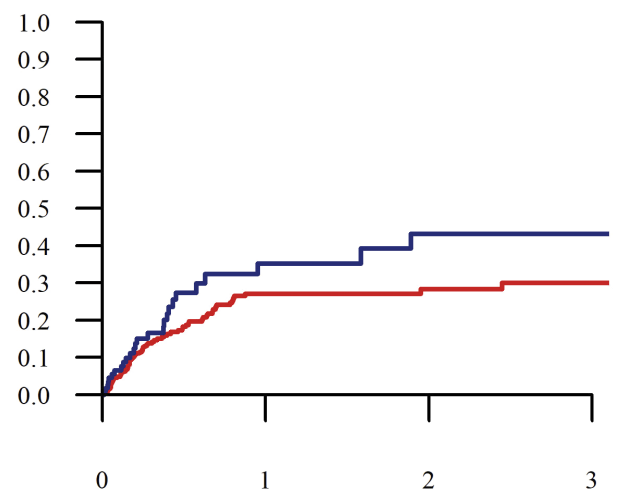

Time from transplant (years)

number of at-risk patients

$\begin{array}{lcccc}\text { ATG } & 98 & 27 & 15 & 11 \\ \text { PTCy } & 336 & 117 & 64 & 30\end{array}$

$c$
0
0
0
0
4
$\frac{0}{0}$
0
0
$\frac{0}{0}$
0

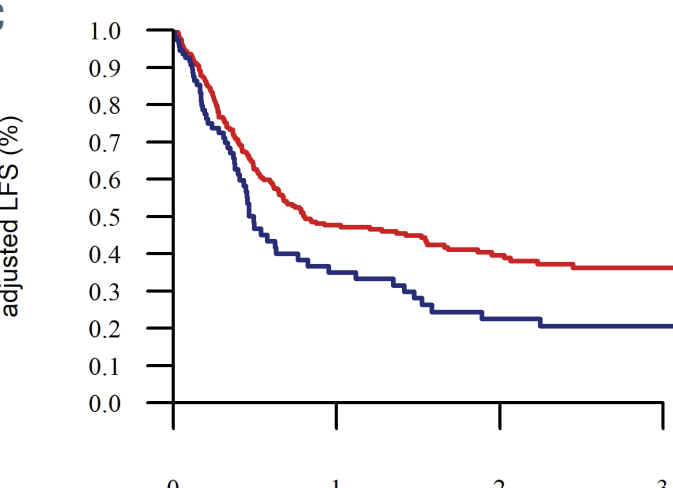

Time from transplant (years) number of at-risk patients

PTCY
27

117
15

64

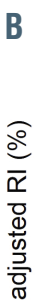

$\begin{array}{lc}\text { ATG } & 98 \\ \text { PTCy } & 336\end{array}$

0

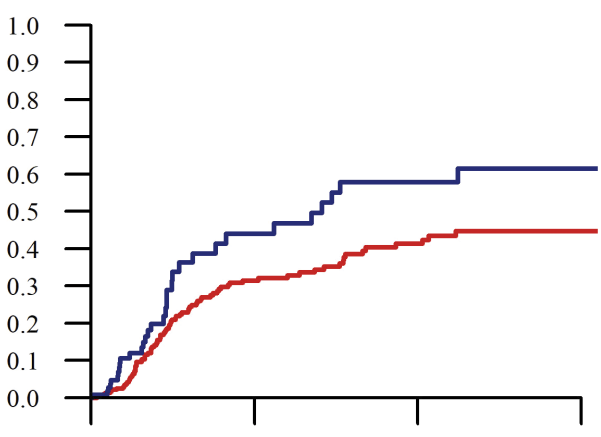

1

2

Time from transplant (years)

number of at-risk patients

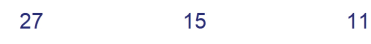

$\begin{array}{lll}117 & 64 & 30\end{array}$

D

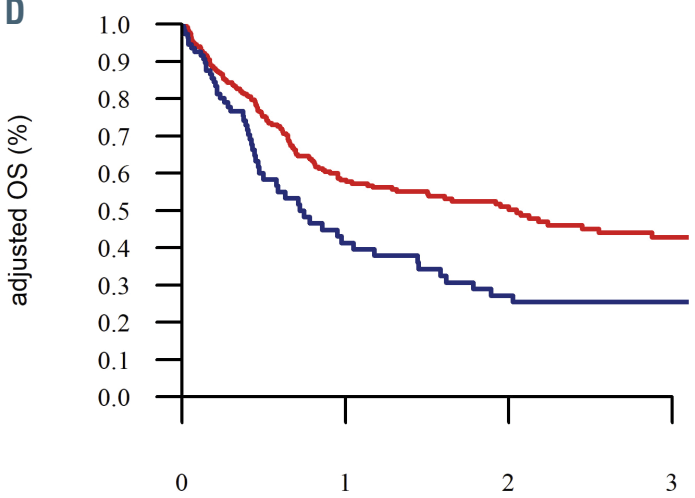

Time from transplant (years)

number of at-risk patients

$\begin{array}{lcccc}\text { ATG } & 98 & 32 & 18 & 14 \\ \text { PTCy } & 336 & 141 & 78 & 36\end{array}$

Figure 1. Adjusted outcomes of patients with acute lymphoblastic leukemia undergoing haploidentical transplantation stratified by type of graft-versus-host disease prophylaxis. Data for recipients of anti-thymocyte globulin are shown in blue, those for recipients of post-transplant cyclophosphamide are shown in red. (A) Adjusted incidence of non-relapse mortality (NRM). (B) Adjusted relapse incidence (RI). (C) Adjusted estimates of leukemia-free survival (LFS), (D) Adjusted estimates of overall survival (OS). 
advanced ALL (HR=3.35, 95\% CI: 2.3-4.88; $P<10^{-5}$ ) were associated with an increased risk of therapy failure, whereas a Karnofsky Performance Score $\geq 90$ was associated with better leukemia-free survival ( $\mathrm{HR}=0.68,95 \% \mathrm{CI}$ : $0.5-0.93 ; P=0.01$ ). Figure $1 \mathrm{C}$ shows the adjusted leukemiafree survival in the ATG and PTCy groups.

The 2-year overall survival rates were $27.4 \%$ (95\% CI: 17.4-37.3) in the ATG group and $48.4 \%$ (95\% CI: $42.3-$ $54.6)$ in the PTCy group $(P=0.001)$ (Table 2, Figure 1D). On multivariate analysis (Table 3), PTCy prophylaxis was associated with better survival compared to ATG prophylaxis (HR=0.6, 95\% CI: $0.42-0.82 ; \quad P=0.003)$. Independently of the GvHD prevention strategy, disease status of second complete remission or beyond ( $\mathrm{HR}=1.88$, 95\% CI: 1.33-2.64; $P=0.0003)$ and advanced ALL (HR=3.13, 95\% CI: 2.15-4.55; $P<10^{-5}$ ) were associated with lower survival, whereas a Karnofsky Performance Score $\geq 90$ was associated with improved overall survival ( $\mathrm{HR}=0.96,95 \% \mathrm{CI}: 0.68-1.38 ; P=0.001)$. Figure $1 \mathrm{D}$ shows adjusted overall survival in the ATG and PTCy groups.

The 2-year GRFS rates in the ATG and PTCy groups were $20 \%$ (95\% CI: $10.9-29.1)$ and $31.8 \%$ (95\% CI: $26.2-$ 37.5), respectively $(P=0.04)$ (Table 2$)$. On multivariate analysis there was no significant difference in GRFS between the ATG and PTCy groups $(P=0.17)$ (Table 3). However, disease status of second complete remission or beyond ( $\mathrm{HR}=1.42,95 \% \mathrm{CI}$ : 1.05-1.92; $P=0.02)$ and advanced ALL (HR=2.45, 95\% CI: $\left.1.73-3.47 ; P<10^{-5}\right)$ were associated with a worse GRFS, whereas a Karnofsky Performance Score $\geq 90$ was associated with a better GRFS (HR=0.77, 95\% CI: 0.58-1.02; $P=0.07$ ).

\section{Causes of death}

At last follow-up, a total of 225 patients had died, including 66 patients in the ATG group (67.3\%) and 159 patients $(47.3 \%)$ in the PTCy group (Table 4). The three most common causes of death in the ATG and PTCy groups were ALL relapse ( $36.5 \%$ vs. $38.1 \%)$, GvHD $(19.1 \%$ vs. $14.2 \%)$ and infections (31.8\% vs. $30.3 \%)$. In the PTCy group, veno-occlusive disease and graft failure was the cause of death in six $(3.9 \%)$ and three $(1.9 \%)$ patients, respectively, while in the ATG group no death was attributed to these. Secondary malignancy as a cause of death was limited to one patient in the entire cohort (in the PTCy group).

\section{Discussion}

GvHD prevention strategies such as ATG, and more recently PTCy, accompanying unmanipulated haploidentical allografts have reinvigorated and ushered in a new era of haploidentical HCT for hematologic malignancies. While ATG is directed against a wide range of epitopes, thus allowing extensive T-cell depletion, ${ }^{24,25}$ PTCy selectively targets alloreactive $\mathrm{T}$ cells rapidly proliferating early after an HLA-mismatched transplant, without affecting the non-dividing hematopoietic progenitor cells. ${ }^{12,26}$ In this study we compared post-transplant outcomes in adult patients with ALL undergoing haploidentical HCT using ATG or PTCy platforms as GvHD prophylaxis and made some important observations. First, no differences in outcome related to acute $\mathrm{GvHD}$, chronic GvHD and non-relapse mortality were seen between the ATG and PTCy groups. Second, relapse risk was signifi-
Table 4. Causes of death in patients with acute lymphoblastic leukemia undergoing haploidentical transplantation with anti-thymocyte globulin or post-transplant cyclophosphamide as graft-versus-host disease prophylaxis.

\begin{tabular}{lcc}
\hline Caluse of Death & ATI $N=66(\%)$ & PICy $\mathbb{N}=159(\%)$ \\
ALL relapse & $23(37.1)$ & $59(38.6)$ \\
Graft-versus-host disease & $12(19.4)$ & $22(14.4)$ \\
\hline Infection & $21(33.9)$ & $49(32.0)$ \\
Veno-occlusive disease & 0 & $6(3.9)$ \\
\hline Failure/rejection & 0 & $3(2.0)$ \\
Cardiac toxicity & $1(1.6)$ & 0 \\
\hline Secondary malignancy & 0 & $2(1.3)$ \\
Hemorrhage & $1(1.6)$ & $4(2.6)$ \\
\hline Interstitial pneumonitis & $2(3.2)$ & $5(3.3)$ \\
Multi-organ failure & $2(3.2)$ & $3(2.0)$ \\
\hline Missing & 4 & 6 \\
\hline
\end{tabular}

ATG: anti-thymocyte globulin; PTCy: post-transplant cyclophosphamide; ALL: acute lymphoblastic leukemia.

cantly higher among patients who received ATG than in those who received PTCy. Third, leukemia-free survival and overall survival were both significantly better in patients treated with PTCy than int those treated with ATG, but there was no difference in GRFS between the groups.

The cumulative incidences of $\mathrm{GvHD}$, including grade II-IV acute GVHD, grade III-IV (severe) acute GvHD and chronic GvHD were similar in the ATG and PTCy groups. The incidence of severe acute GvHD in the ATG group $(11.6 \%)$ is comparable to the incidences in previous observational studies. ${ }^{11,25}$ Interestingly, in the ALL-specific prospective study by Wang et al., the incidence of severe acute GvHD was only $6 \%$ in the group that underwent haploidentical HCT with ATG prophylaxis. ${ }^{27}$ The incidence of severe acute GvHD in the PTCy group in this study $(14.6 \%)$ is higher than the previously reported 4 $5 \% .^{12,15}$ The higher incidence of acute GvHD may be related to the substantial proportions of patients in this study who were given myeloablative conditioning $(76 \%)$, TBI (41\%), and peripheral blood products $(52 \%)$ and had advanced disease $(20 \%)$ at the time of HCT, compared to the proportions in other studies. However, the incidences of chronic GvHD in the ATG (28\%) and PTCy (32\%) groups are comparable to those in prior reports. ${ }^{11,25,27}$ It is noteworthy that additional immunosuppression in the ATG group consisted predominantly of cyclosporine/ methotrexate/mycophenolate $(42.5 \%)$ or sirolimus/ mycophenolate $(22.3 \%)$, whereas in the PTCy group $85 \%$ of the patients received a calcineurin inhibitor (cyclosporine or tacrolimus) with mycophenolate (Table 1).

The incidences of non-relapse mortality in the ATG and PTCy groups were similar (33\% vs. $27 \%$, respectively), likely reflecting the similarity in GvHD incidences as noted above. The major causes of non-relapse mortality in both groups were GvHD and infection. Interestingly, in the PTCy group, six patients died of veno-occlusive disease presumably related to myeloablative conditioning with TBI, and three patients from graft failure likely due to more bone marrow grafts being used in this group. However, on multivariate analysis, only advanced disease state (relapsed beyond second complete remission and refractory ALL) was an independent predictor of poor 
non-relapse mortality. Although most patients received albative doses of TBI, a lower non-relapse mortality was associated with TBI-based condtioning compared to chemotherapy alone ( $\mathrm{HR}=0.59 ; P=0.02)$. An explanation for this intriguing observation is unknown, but it is possible that younger patients with better performance scores and co-morbidity indices were more likely to receive ablative TBI. It should be noted that previous EBMT reports have indeed suggested better leukemia-free survival related to TBI use in ALL patients, but this was due to a lower relapse incidence and not to a decrease in nonrelapse mortality. $28-30$

With respect to post-transplant relapse, a statistically significant, $10 \%$ absolute improvement was noted in favor of PTCy compared to the ATG group. It is likely that the higher risk of relapse in the ATG group was driven by the higher proportion of patients with advanced ALL (30\%). Indeed, advanced disease status was a strong independent predictor of ALL relapse. Given the unique biology of ALL it is also possible that, as fewer patients given ATG received TBI as part of their conditioning, this may have increased the relapse incidence in this group, although not to a statistically significant extent. ${ }^{28,29,31}$ Disease relapse was the most common cause of death in both groups, highlighting the need for further studies on mitigating post-transplant ALL relapse.

In univariate analysis, improved leukemia-free survival, overall survival and GRFS rates were noted in the PTCy group compared to ATG group $(P<0.05)$, however, in multivariate analysis, only leukemia-free survival and overall survival remained significantly improved. This could perhaps be due to a higher proportion of patients with advanced disease together with the higher relapse incidence in the ATG group. Indeed, the leukemia-free survival in the ATG group in this study was inferior to that in the prospective study by Wang et al., possibly due to the greater proportion of patients in this study with advanced age and disease status. ${ }^{27}$ Nevertheless, $15 \%$ and $20 \%$ improvements in leukemia-free survival and overall survival, respectively, were noted in the PTCy group. For both these outcome measures, disease status at the time of haploidentical HCT and Karnofsky Performance Score were strong independent predictors of survival. Albeit limited by the small sample size, a subset analysis evaluating outcomes stratified by graft source (peripheral blood vs. bone marrow) showed interesting results (Online Supplementary Table S2). In peripheral blood haploidentical HCT recipients, the 2-year leukemia-free survival, overall survival and GRFS rates were significantly better in the PTCy group, in which there was also a trend toward lower non-relapse mortality. It is worth noting that the relapse risk was similar in the ATG and PTCy groups in peripheral blood haploidentical graft recipients. In contrast, for bone marrow haploidentical HCT, no differences were noted in leukemia-free survival, overall survival, GRFS or non-relapse mortality between the ATG and PTCy groups. However, the 2-year relapse incidence among bone marrow graft recipients receiving ATG prophylaxis was $55 \%$ compared to $33.7 \%$ with PTCy prophylaxis $(P=0.06)$. Although not statistically significant, it is plausible that the inferior relapse and survival outcomes in the ATG group are at least partly due to the high relapse risk in the bone marrow graft recipients..$^{32}$

The inherent limitations of this study are reflected by the nature of the data captured by a registry. Detailed information regarding remission status, such as minimal residual disease, and conditioning regimen, including TBI dose and the timing and dose of PTCy and ATG administration, were not uniformly available. For instance, details pertaining to the type of ATG product (thymoglobulin vs. ATG-Fresenius) were unavailable in the registry. The dose of ATG was documented for only 81 subjects, with the median dose being $20 \mathrm{mg} / \mathrm{kg}$. By univariate analysis, the only impact of ATG dose $(<$ or $\geq 20 \mathrm{~g} / \mathrm{kg}$ ) on transplant outcomes, was a lower incidence of grade II-IV acute GvHD associated with a higher ATG dose $(23 \%$ vs. $53 \%$; $P=0.007$ ) (Online Supplementary Table S3). The registry data precluded evaluation of the reason for choosing a specific graft source, GvHD prophylaxis platform or conditioning regimen for an individual patient. Haploidentical HCT with ATG was more likely during an earlier time period compared to PTCy (median, 2011 vs. 2015). It is, therefore, possible that improvements in transplant technology and supportive care may have had an impact on these outcomes. To address this potential bias, we performed a univariate analysis restricted to the years 2007-2014, and found that the use of PTCy as GvHD prophylaxis was still associated with improved leukemia-free and overall survival (data not shown), congruent with results for the entire study duration. As expected, institutional practices and preferences may skew the data. However, no "center effect" was noted except for chronic GvHD by regression analysis. The sample size limited the power to detect small differences and interactions between variables and transplant outcomes in our population. With a median follow-up of approximately 2 years, it is not known whether these results will remain unchanged with longer-term follow-up. Notwithstanding these limitations, this analysis is the largest and only comparative study evaluating outcomes of haploidentical allogeneic HCT in adult ALL patients given PTCy or ATG as the backbone of their GvHD prophylaxis. It is noteworthy that, compared to ATG, PTCy as GvHD prophylaxis was associated with improved leukemia-free survival and overall survival and lower relapse risk.

In conclusion, in the absence of prospective, randomized data, our results suggest that PTCy as GvHD prophylaxis may be considered over ATG in patients with ALL undergoing haploidentical HCT. Our data warrant confirmation in prospective randomized studies.

\section{Disclosures}

No conflicts of interest to disclose.

\section{Contributions}

$A N$ and $M M$ conceived and designed the study; ML collected, assembled and analyzed data; $A S K, A N$ and ML prepared the first draft of the manuscript; and all authors contributed to data interpretation, helped revise the manuscript, and gave final approval of the manuscript. 


\section{References}

1. Juliusson G, Karlsson K, Lazarevic VL, et al. Hematopoietic stem cell transplantation rates and long-term survival in acute myeloid and lymphoblastic leukemia. Cancer. 2011;117(18):4238-4246.

2. Fielding AK, Richards SM, Chopra R, et al. Outcome of 609 adults after relapse of acute lymphoblastic leukemia (ALL); an MRC UKALL12/ECOG 2993 study. Blood. 2007; 109(3):944.

3. Gupta V, Richards S, Rowe J. Allogeneic, but not autologous, hematopoietic cell transplantation improves survival only among younger adults with acute lymphoblastic leukemia in first remission: an individual patient data meta-analysis. Blood. 2013; 121(2):339

4. D'Souza A, Lee S, Zhu X, Pasquini M. Current use and trends in hematopoietic cell transplantation in the United States. Biol Blood Marrow Transplant. 2017;23(9):14171421.

5. Mancusi A, Ruggeri L, Velardi A. Haploidentical hematopoietic transplantation for the cure of leukemia: from its biology to clinical translation. Blood. 2016;128 (23):2616.

6. Henslee-Downey P, Parrish R, MacDonald J, et al. Combined in vitro and in vivo T lymphocyte depletion for the control of graftversus-host disease following haploidentical marrow transplant. Transplantation. 1996; 61(5):738-745.

7. Dodero A, Carniti C, Raganato A, et al. Haploidentical stem cell transplantation after a reduced-intensity conditioning regimen for the treatment of advanced hematologic malignancies: posttransplantation CD8-depleted donor lymphocyte infusions contribute to improve T-cell recovery. Blood. 2009;113(19):4771-4779

8. Kanda Y, Oshima K, Asano-Mori Y, et al. Invivo alemtuzumab enables haploidentical human leukocyte antigen-mismatched hematopoietic stem-cell transplantation without ex vivo graft manipulation. Transplantation. 2005;79(10):1351-1357.

9. Aversa F, Terenzi A, Tabilio A, et al. Full haplotype-mismatched hematopoietic stem-cell transplantation: a phase II study in patients with acute leukemia at high risk of relapse. J Clin Oncol. 2005;23(15):3447-3454.

10. Lu D-P, Dong L, Wu T, et al. Conditioning including antithymocyte globulin followed by unmanipulated HLA-mismatched/haploidentical blood and marrow transplantation can achieve comparable outcomes with HLA-identical sibling transplantation. Blood. 2006;107(8):3065.
11. Wang Y, Liu D-H, Liu K-Y, et al. Long-term follow-up of haploidentical hematopoietic stem cell transplantation without in vitro $\mathrm{T}$ cell depletion for the treatment of leukemia. Cancer. 2013;119(5):978-985

12. Luznik L, Jalla S, Engstrom L, Iannone R, Fuchs $\mathrm{E}$. Durable engraftment of major histocompatibility complex-incompatible cells after nonmyeloablative conditioning with fludarabine, low-dose total body irradiation, and posttransplantation cyclophosphamide. Blood. 2001;98(12):3456-3464.

13. O'Donnell P, Luznik L, Jones $\mathrm{R}$, et al. Nonmyeloablative bone marrow transplantation from partially HLA-mismatched related donors using posttransplantation cyclophosphamide. Biol Blood Marrow Transplant. 2002;8(7):377-386.

14. Santoro N, Ruggeri A, Labopin M, et al. Unmanipulated haploidentical stem cell transplantation in adults with acute lymphoblastic leukemia: a study on behalf of the Acute Leukemia Working Party of the EBMT. J Hematol Oncol. 2017;10(1):113.

15. Srour SA, Milton DR, Bashey A, et al. Haploidentical transplantation with posttransplantation cyclophosphamide for highrisk acute lymphoblastic leukemia. Biol Blood Marrow Transplant. 2017;23(2):318-324.

16. Iskra Pusic, Steven Z. Pavletic. Challenges in conducting studies in chronic graft-versushost disease. Clin Hematol Int. 2019;1(1):3644

17. Holtan SG, DeFor TE, Lazaryan A, et al. Composite end point of graft-versus-host disease-free, relapse-free survival after allogeneic hematopoietic cell transplantation. Blood. 2015;125(8):1333-1338.

18. Ruggeri A, Labopin M, Ciceri F, Mohty M, Nagler A. Definition of GvHD-free, relapsefree survival for registry-based studies: an ALWP-EBMT analysis on patients with AML in remission. Bone Marrow Transplantation. 2015;51(4):610.

19. Przepiorka D, Weisdorf D, Martin P, et al 1994 Consensus Conference on Acute GVHD Grading. Bone Marrow Transplant. 1995;15(6):825-828.

20. Lee SJ, Vogelsang G, Flowers MED. Chronic graft-versus-host disease. Biol Blood Marrow Transplant. 2003;9(4):215-233.

21. Bacigalupo A, Ballen K, Rizzo D, et al. Defining the intensity of conditioning regimens: working definitions. Biol Blood Marrow Transplant. 2009;15(12):1628-1633.

22. Hougaard P. Frailty models for survival data. Lifetime Data Anal. 1995;1(3):255-273.

23. Andersen PK, Klein JP, Zhang M-J. Testing for centre effects in multi-centre survival studies: a Monte Carlo comparison of fixed and random effects tests. Stat Med. 1999;18(12):1489-1500.
24. Mohty M, Bacigalupo A, Saliba F, et al. New directions for rabbit antithymocyte globulin (Thymoglobulin(®)) in solid organ transplants, stem cell transplants and autoimmunity. Drugs. 2014;74(14):1605-1634.

25. Ruggeri A, Sun Y, Labopin M, et al. Posttransplant cyclophosphamide versus antithymocyte globulin as graft- versus-host disease prophylaxis in haploidentical transplant. Haematologica. 2017;102(2):401.

26. Kanakry C, Ganguly S, Zahurak M, et al. Aldehyde dehydrogenase expression drives human regulatory $\mathrm{T}$ cell resistance to posttransplantation cyclophosphamide. Sci Transl Med. 2013;5(211):211ra157.

27. Wang Y, Liu O-F, Xu L-P, et al. Haploidentical versus matched-sibling transplant in adults with Philadelphia-negative high-risk acute lymphoblastic leukemia: a biologically phase III randomized study. Clin Cancer Res. 2016;22(14):3467.

28. Giebel S, Labopin M, Socié G, et al Improving results of allogeneic hematopoietic cell transplantation for adults with acute lymphoblastic leukemia in first complete remission: an analysis from the Acute Leukemia Working Party of the European Society for Blood and Marrow Transplantation. Haematologica. 2017;102 (1):139-149

29. Pavlů J, Labopin M, Zoellner AK, et al. Allogeneic hematopoietic cell transplantation for primary refractory acute lymphoblastic leukemia: a report from the Acute Leukemia Working Party of the EBMT. Cancer. 2017;123(11):1965-1970.

30. Rashmika R. Potdar, Sorab Gupta, Sebastian Giebel, et al. Current status and perspectives of irradiation-based conditioning regimens for patients with acute leukemia undergoing hematopoietic stem cell transplantation. Clin Hematol Int. 2019;1(1):19-27.

31. Eder S, Canaani J, Beohou E, et al. Thiotepabased conditioning versus total body irradiation as myeloablative conditioning prior to allogeneic stem cell transplantation for acute lymphoblastic leukemia: a matched-pair analysis from the Acute Leukemia Working Party of the European Society for Blood and Marrow Transplantation. Am J Hematol. 2017;92(10):997-1003

32. Nagler A, Labopin M, Shimoni A, et al Mobilized peripheral blood stem cells compared with bone marrow as the stem cell source for unrelated donor allogeneic transplantation with reduced-intensity conditioning in patients with acute myeloid leukemia in complete remission: an analysis from the Acute Leukemia Working Party of the European Group for Blood and Marrow Transplantation. Biol Blood Marrow Transplant. 2012;18(9):1422-1429. 